\title{
Synergistic Effects of SAM and Selenium Compounds on Proliferation, Migration and Adhesion of HeLa Cells
}

\author{
LICUI SUN $^{1 *}$, JIANXIN ZHANG ${ }^{2 *}$, QIU YANG ${ }^{1}$, YANG SI ${ }^{3}$, YIQUN LIU ${ }^{1}$, \\ QIN WANG ${ }^{1}$, FENG HAN ${ }^{1}$ and ZHENWU HUANG ${ }^{1}$ \\ ${ }^{1}$ Department of Nutrition and Metabolism, National Institute for Nutrition and Health, \\ Chinese Center for Disease Control and Prevention, Beijing, P.R. China; \\ ${ }^{2}$ Department of Gynecology, Beijing Chaoyang Hospital Affiliated to Capital Medical University, Beijing, P.R. China; \\ ${ }^{3}$ Department of Biochemistry and Molecular Biology, Capital Medical University, Beijing, P.R. China
}

\begin{abstract}
Background/Aim: To determine the antitumor activities and molecular mechanism of selenium compounds in HeLa cells. Materials and Methods: Western blotting was used to detect ERK and AKT activation in HeLa cells induced by selenium compounds selenomethionine (SeMet), methylselenocysteine (MeSeCys) and methylseleninic acids (MeSeA). Using MTT, wound-healing and Matrigel adhesion assays, the antitumor effects of SAM and selenium compounds were evaluated in HeLa cells. Results: MeSeA inhibited ERK and $A K T$ signaling pathways and suppressed the proliferation $(p<0.05$ vs. HeLa control), migration ( $p<0.05$ vs. HeLa control) and adhesion ( $p<0.01 \mathrm{vs.} \mathrm{HeLa} \mathrm{control)} \mathrm{of} \mathrm{HeLa} \mathrm{cells.}$ MeSeCys and SeMet inhibited AKT signaling pathways and the migration ( $p<0.05$ vs. HeLa control) and adhesion ( $p<0.01 v \mathrm{vs.}$ $\mathrm{HeLa}$ control) of HeLa cells. The synergistic action of MeSeA with SAM led to a statistically significant inhibition of proliferation, migration and adhesion of $\mathrm{HeLa}$ cells. Conclusion: MeSeA, MeSeCys and SeMet exert different antitumor activities by inhibiting ERK and AKT signaling pathways. The combination of MeSeA and SAM exhibited better antitumor effects compared to the other treatments.
\end{abstract}

Selenium is an essential trace element for normal bodily function. Thus, selenium deficiency is associated with disease conditions, such as Keshan disease, and general

\footnotetext{
*These Authors contributed equally to this study.

Correspondence to: Zhenwu Huang, Department of Nutrition and Metabolism, National Institute for Nutrition and Health, Chinese Center for Disease Control and Prevention, 29 Nanwei Road, Xicheng District, Beijing 100050, P.R. China. Tel: +86 1066237224 Fax: +86 1083132021, e-mail: zhenwuhuang@sina.com

Key Words: SAM, selenium compounds, signaling pathways, synergistic effects.
}

impairment of the immune system. Currently a large number of human trials and animal experiments indicate that increased nutritional levels of selenium can exert cancer chemo-preventive effects $(1,2)$. Meta-analysis showed that selenium decreased the risk of occurrence of prostate cancer, bladder cancer, etc., demonstrating potential antitumor effects $(3,4)$.

The antitumor effects of selenium supplementation depend on a number of factors including chemical forms and the administered dosage both of which appear to be key influences. Selenomethionine (SeMet), methylselenocysteine (MeSeCys) and methylseleninic acids (MeSeA) are three main forms of organic selenium, which are present as selenoamino acids. SeMet and MeSeCys are typical naturally found nutrition selenium sources. SeMet is the main form of dietary selenium that is mainly found in grains and beans whilst MeSeCys is the main form of selenium found in garlic and onion. MeSeA is a chemically synthesized inorganic selenium compound.

Studies show that SeMet, MeSeCys and MeSeA exert different antitumor activities. At in vivo level, oral MeSeA treatment of xenograft models inhibited tumor growth in a dose-dependent manner. Furthermore, MeSeA demonstrated superior inhibitory efficacy on in vivo growth compared to SeMet in two human prostate cancer (PCa) xenograft models (5). MeSeCys is an organoselenium compound being developed for breast cancer chemoprevention (6). MeSeCys can inhibit proliferation and induce apoptosis and $\mathrm{S}$ phase arrest in MDA-MB-231 cells (7). In the animal model of colon cancer, MeSeCys can inhibit tumor angiogenesis and growth of colon cancer (8). The above studies have shown that $\mathrm{MeSeA}$ and MeSeCys have superior antitumor effects. SeMet, as the major component of dietary selenium, has been used for cancer chemoprevention in several clinical trials $(9,10)$. However, evidence from experimental studies has indicated that SeMet has weaker antitumor effects than most other forms of selenium (11). In A549 lung carcinoma cells, SeMet caused 
ROS-dependent apoptosis (12). Whilst in human colon cancer cells SeMet induced sustained ERK phosphorylation leading to cell-cycle arrest (13). Yet in gastric cancer cells, SeMet stimulated MAPK (ERK) phosphorylation, protein oxidation, and DNA synthesis (14), exhibiting tumor promoting effects. The above studies highlight that the antitumor effects of SeMet remain controversial.

S-adenosyl-methionine (SAM) is a universal methyl group donor to various intermediary metabolites. Multiple clinical trials have indicated that SAM is important in the treatment of Alzheimer's disease, depression and osteoarthritis $(15,16)$. However, therapeutic usages of SAM are not yet proven in cancer. Recent studies show SAM has antitumor activity in some cancer tissues and cells. In colorectal cancer cells, SAM inhibited the proliferation and bio-energetic function (17). Further evidence has shown that SAM inhibited azoxymethaneinduced colonic aberrant crypt foci in F344 rats and suppressed human colon cancer Caco-2 cell growth in 3D culture (18). SAM specifically protected the anticancer effect of 5-FU in human A549 lung cancer cells (19). Treatment with SAM resulted in a dose-dependent inhibition of osteosarcoma cells proliferation, invasion, cell migration, and cell cycle (20). Selenide and SAM could react spontaneously to form methylselenol, which is the key active antitumor substance (21). However, the molecular mechanism of this remains unknown.

In this study, we screened for selenium compounds-related signaling pathways of $\mathrm{HeLa}$ cells and evaluated the individual and synergistic antitumor effects of SAM and selenium compounds in HeLa cells using a variety of in vitro techniques. For the first time SAM was found to synergistically inhibit the proliferation, migration and adhesion of HeLa cells caused by selenium compounds by inactivating the ERK and AKT signaling pathways.

\section{Materials and Methods}

Chemicals. The selenium compounds MeSeA, SeMet and MeSeCys were purchased from Sigma (St. Louis, MO, USA). SAM and crystal violet were also purchased from Sigma. Matrigel was purchased from BD (Franklin Lakes, NJ, USA). MTT detection kit was purchased from Merck (Darmstadt, Hessen, Germany). Horseradish peroxidase-conjugated anti-mouse and anti-rabbit secondary antibodies were purchased from Amersham Biosciences (Piscataway, NJ, USA). Total ERK and AKT primary antibodies and the primary antibodies for phospho-ERK1/2 (pERK) and phosphoAKT (pAKT) were purchased from Cell Signaling Technology (Beverly, MA, USA). Anti-GAPDH antibody was purchased from Cell Signaling Technology (Beverly, MA, USA). All reagents were of analytical grade or the highest grade available.

Cell culture and treatment. Human cervical carcinoma cell line HeLa was purchased from the American Type Culture Collection (ATCC, Manassas, VA, USA). HeLa cells were cultured in DMEM plus $10 \%$ FBS, $1 \%$ penicillin and streptomycin (Gibco, Paisley, Scotland, UK). All cells were respectively treated with different concentrations of selenium compounds $(0.3-30 \mu \mathrm{M})$ and/or SAM $(3 \mu \mathrm{M})$ for $12-72 \mathrm{~h}$ at $37^{\circ} \mathrm{C}$ and incubated in $5 \% \mathrm{CO}_{2}$. HeLa cells without treatment served as a control group.

Western blotting. Western blotting was performed to probe for phospho-ERK1/2 and phospho-AKT expression levels. In brief, sample aliquots corresponding to $25 \mu \mathrm{g}$ of protein were resolved using $10 \%$ sodium dodecyl sulfate-polyacrylamide gel electrophoresis (SDS-PAGE) for $1 \mathrm{~h}$ at $150 \mathrm{~V}$ and then transferred to a nitrocellulose membrane. The blots were blocked in the blocking buffer (5\% nonfat dry milk in TBST) for at least $30 \mathrm{~min}$ and then incubated with primary antibody in TBST $(20 \mathrm{mM}$ Tris $/ \mathrm{HCl}, \mathrm{pH} 7.5,500 \mathrm{mM} \mathrm{NaCl}, 0.05 \%$ Tween 20) for $1 \mathrm{~h}$ at room temperature or overnight at $4^{\circ} \mathrm{C}$. The blots were then washed three times with $10 \mathrm{ml}$ of TBST each and incubated for $30 \mathrm{~min}$ at room temperature with a horseradish peroxidase-conjugated secondary antibody in TBST. Finally, the blots were washed three more times with $10 \mathrm{ml}$ of TBST each and visualized by enzyme-linked chemiluminescence (Amersham Biosciences, NJ, USA). The phospho-ERK and phospho-AKT were probed. Protein levels were normalized with GAPDH, and the levels of phospho-ERK and phospho-AKT immunoreactivities were normalized to the total ERK and total AKT immunoreactivities. Immunoreactive bands were quantified by NIH Image 1.62 (National Institutes of Health, Bethesda, MD, USA).

In vitro tumor cell growth assay (Cell proliferation assay). Cells were seeded into a 96-well plate (Nunc, Fisher Scientific, Leicestershire, UK) at a seeding density of $2.5 \times 10^{3}$ cells in $200 \mu \mathrm{l}$ medium with addition of selenium compounds $(3 \mu \mathrm{M})$ and/or SAM $(3 \mu \mathrm{M})$ per well. $12 \mathrm{~h}$ after incubation, cells were added with $50 \mu \mathrm{l}$ MTT $(2 \mathrm{mg} / \mathrm{ml}$ of stock solution) at indicated time points and cultured for $3 \mathrm{~h}$. Then $150 \mu \mathrm{l}$ DMSO was used to dissolve crystallization and viable cells were quantified by measuring absorbance at $490 \mathrm{~nm}$ with an Elx800 ${ }^{\mathrm{TM}}$ spectrophotometer (BioTek, Winooski, VT, USA).

In vitro tumor cell migration assay (Wound-healing assay). The wound-healing assay was used to assess the migratory properties of the cells. Cells were seeded at a density of $2 \times 10^{5}$ cells/well into a 24-well plate, added with $3 \mu \mathrm{M}$ of selenium compounds and/or $3 \mu \mathrm{M}$ of SAM, and allowed to reach confluence. The layer of cells was then scraped with a fine gauge needle to create a wound of approximately $200 \mu \mathrm{m}$. The movement of cells to heal the wound was recorded and analyzed using a time-lapsed video system. Cell migration was measured using Image $\mathbf{J}$ analysis software.

The distance between the two wound fronts at 10 random points per incubation time was calculated using the Image J software; the arbitrary values obtained were converted into $\mu \mathrm{m}$ by multiplying the value of 0.82 . The distance that the wound fronts had migrated into the wound at each time point could then be determined by subtracting the distance between the two fronts at any given time point from that at the start point.

In vitro tumor cell Matrigel adhesion assay. The ability of tumor cells to adhere to an artificial Matrigel basement membrane was examined using an in vitro Matrigel adhesion assay. $100 \mu \mathrm{l}$ of serum-free medium including $5 \%(\mathrm{w} / \mathrm{v})$ Matrigel was added to each well of a 96-well plate and dried in an oven to form an artificial basement membrane. The membrane was then rehydrated in $100 \mu \mathrm{l}$ 
of serum-free medium for $40 \mathrm{~min}$ before cell seeding. $4.5 \times 10^{4}$ cells were seeded onto the Matrigel basement membrane in $200 \mu \mathrm{l}$ of normal medium and incubated for $40 \mathrm{~min}$. Following incubation, the medium was removed and the membrane was washed five times with $150 \mu \mathrm{l}$ of BSS solution to remove only loosely attached cells. Adherent cells were then fixed in $4 \%$ formaldehyde (v/v) in BSS for $15 \mathrm{~min}$ before being stained in $0.5 \%$ crystal violet solution $(\mathrm{w} / \mathrm{v})$ for $5 \mathrm{~min}$. The stain was then extracted from the cells using $150 \mu \mathrm{l}$ of $10 \%$ glacial acetic acid and cell density was determined by measuring the absorbance at $540 \mathrm{~nm}$ on a microplate reader.

Statistical analysis. All experiments were repeated at least three times. The data are presented as the means \pm S.D. Data were analyzed using SPSS 18.0 statistical software and 2-sample $t$-test. $p<0.05$ was considered to be statistically significant.

\section{Results}

Dose determination of MeSeA affected HeLa cells proliferation. To determine the dose effects of selenium compounds on tumor cell function, HeLa cells were treated with $0.3 \mu \mathrm{M}, 1 \mu \mathrm{M}, 3 \mu \mathrm{M}, 10 \mu \mathrm{M}$ and $30 \mu \mathrm{M}$ MeSeA for 24-72 h. MTT assay was used to assess HeLa cell proliferation. Results showed that cell proliferation was inhibited in a dose dependent manner at 24, 48 and $72 \mathrm{~h}$ (Figure 1) in MeSeA-treated HeLa cells, though none of these changes reached statistical significance. The doses of $10 \mu \mathrm{M}$ and $30 \mu \mathrm{M}$ MeSeA caused cell death. Therefore, the dose of $3 \mu \mathrm{M}$ of $\mathrm{MeSeA}$, the maximum effective dose, was used to determine phospho-ERK and phospho-AKT and evaluate cell proliferation, migration and adhesion of HeLa cells in the following experiments.

Combining selenium compounds with SAM inhibits ERK and AKT activation in HeLa cells. Selenium compounds have been reported to be involved in the regulation of AKT and ERK signaling pathways, which in turn control important biological functions such as cell proliferation and migration. Therefore, the possibility of combining selenium compounds with SAM affecting HeLa cell functions via the ERK and AKT pathways was investigated. The results are shown in Figure 2. MeSeA alone or combined with SAM significantly inhibited both ERK and AKT activation. The level of ERK (pERK) and AKT activation (pAKT) decreased by $65 \%$ and $55 \%$ in HeLa cells treated with MeSeA $(* * p<0.01, \# p<0.05)$. The levels of pERK and pAKT decreased by $80 \%$ and $70 \%$ in cells treated with MeSeA combining SAM $(* * p<0.01$, $\# p<0.05$ ), respectively. Compared to MeSeA alone, MeSeA combining SAM significantly suppressed pERK and pAKT level about $20 \%$ and $15 \%$, with statistical significance $(\Delta / \gamma$ $p<0.05$ ) (Figure 2A). SeMet alone or combined with SAM significantly inhibited AKT activation by $45 \%$ and $50 \%$ $(\# p<0.05)$. The presence of SAM had no obvious effects on SeMet-inhibited pAKT level (Figure 2B). MeSeCys alone or combined with MeSeCys significantly inhibited AKT

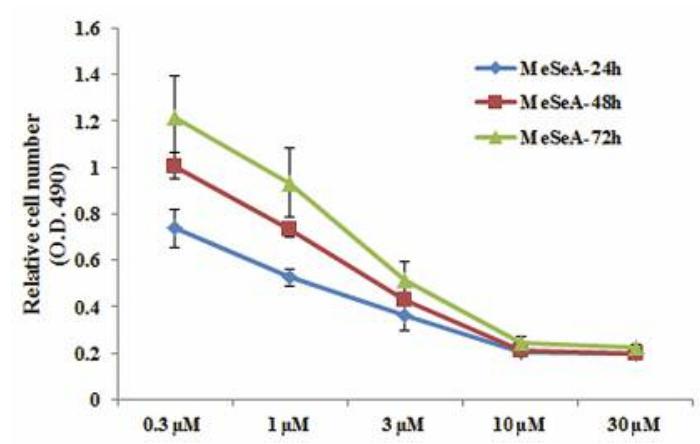

Figure 1. The dose curve of MeSeA affected HeLa cells proliferation. HeLa cells were exposed to MeSeA at a concentration of $0.3,1,3,10$ and $30 \mu \mathrm{M}$ MeSeA for 24, 48 and $72 \mathrm{~h}$, respectively. MTT assay was used to detect the proliferation of HeLa cells. The dose of $3 \mu \mathrm{M}$ of $\mathrm{MeSeA}$ was found to be as the maximum effective dose. The results shown are representative of three individual experiments. Data are mean $\pm S . D$.

activation by $25 \%$ and $45 \% \quad(\# p<0.05)$. Compared to MeSeCys alone, MeSeCys combined with SAM significantly suppressed pAKT levels about 20\%, with statistical significance $(\gamma p<0.05)$ (Figure $2 \mathrm{C})$. Also, SAM was found to significantly suppress pERK level $(* p<0.05)$. Meanwhile the total AKT and ERK remained unchanged. These data indicate that selenium compounds and SAM synergistically inhibit ERK and AKT activation in HeLa cells.

Combining MeSeA with SAM results in a significant proliferation inhibition in HeLa cells. Major malignant phenotypes of tumor cells include cell proliferation, migration, invasion and adhesion. MTT assay was first used to assess the HeLa cells proliferation by combining selenium compounds with SAM. Compared with HeLa control cells, the cell proliferation was significantly suppressed in MeSeAtreated HeLa cells about $30 \%$, with statistical significance at $36,48,60$ and $72 \mathrm{~h}$ individually $(p<0.05)$ (Figure 3A). The treatment of SAM also resulted in the proliferation inhibition of HeLa cells, with statistical significance at the time point of 60 and $72 \mathrm{~h}$ individually $(p<0.05)$. Compared with MeSeA or SAM alone, MeSeA combining SAM significantly suppressed HeLa cells proliferation about $25 \%$, with statistical significance at 60 and $72 \mathrm{~h}$ individually $(p<0.05)$ (Figure 3B). SeMet in the presence or absence of SAM had no obvious effects on HeLa cells proliferation (Figure 3C). MeSeCys also had no obvious effects (Figure 3D). These data indicate that MeSeA and SAM synergistically inhibited the proliferation of HeLa cells.

Selenium compounds and SAM synergistically inhibit the migration of HeLa cells. To further investigate if selenium compounds and SAM could synergistically affect the 

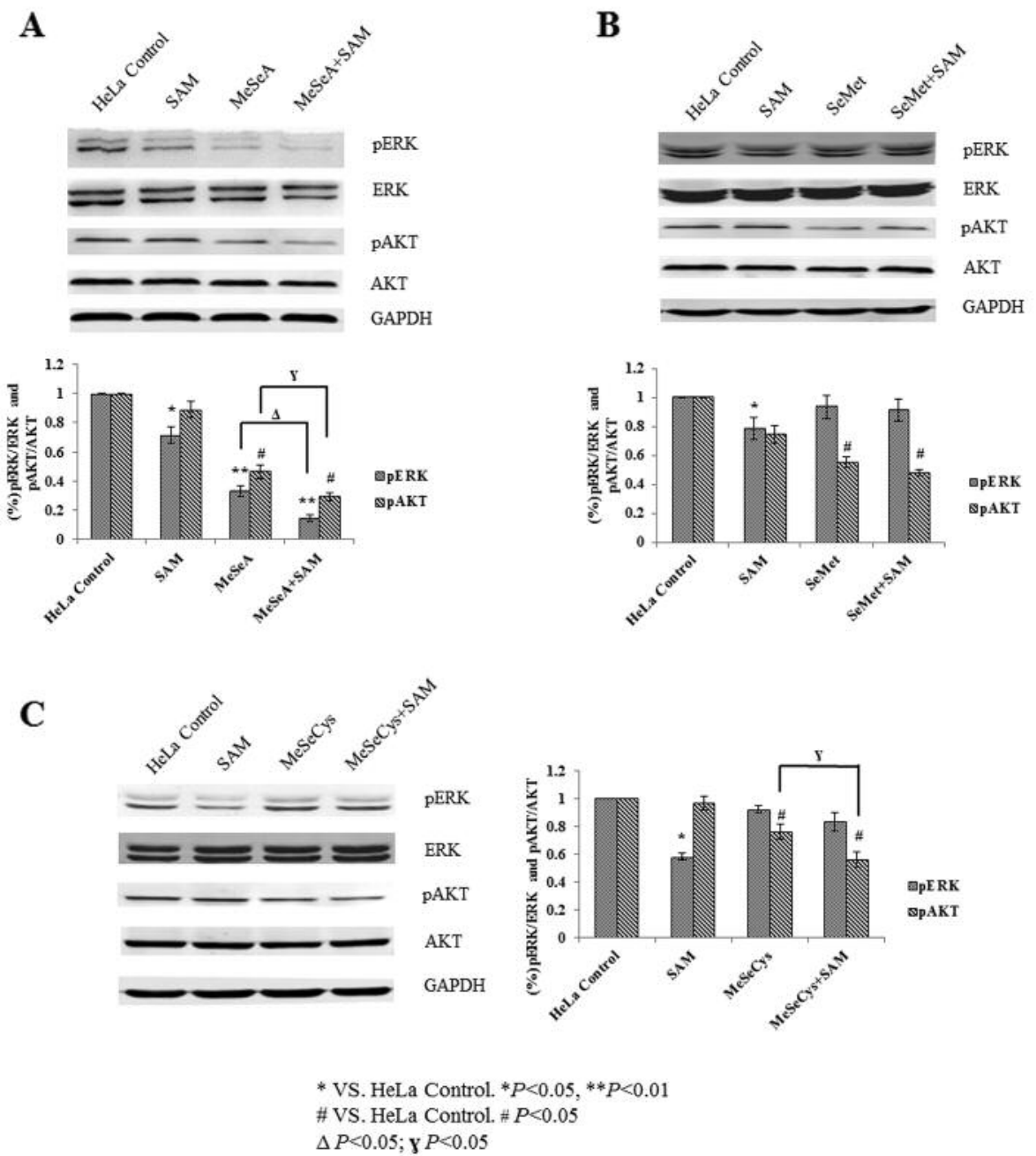

Figure 2. Combining selenium compounds with SAM inhibits ERK and AKT activation in HeLa cells. HeLa cells were treated with $3 \mu M$ of selenium compounds (MeSeA, MeSeCys and SeMet) and/or $3 \mu \mathrm{M}$ of SAM for 24 h, starved for 24 h following 15 min stimulation. Western blotting was performed to probe for phospho-ERK and phospho-AKT. GAPDH was used as a loading control. (A) Compared to HeLa control cells, MeSeA alone or combined with SAM significantly inhibited both ERK and AKT activation. Compared with MeSeA alone, MeSeA combining SAM significantly suppressed pERK and pAKT level. (B) Compared with HeLa control cells, SeMet alone or combined with SAM significantly inhibited AKT activation. (C) Compared to HeLa control cells, MeSeCys alone or combined with MeSeCys significantly inhibited AKT activation. Compared with MeSeCys alone, MeSeCys combining SAM significantly suppressed pAKT level. Data are representative of 3 independent experiments ( $n=3$ ). Data are mean $\pm S . D . *$, \#p<0.05 vs. HeLa control. $4, \gamma$ p $<0.05$ vs. without SAM.

migration of tumor cells, the wound-healing assay was used. Results are shown in Figure 4. Compared to HeLa control cells, the treatment of MeSeA, SeMet or MeSeCys all inhibited the migration of HeLa cells. The migration inhibition rate of MeSeA, SeMet or MeSeCys in $4 \mathrm{~h}$ was $40 \%, 30 \%$ and $20 \%$, respectively, and $40 \%, 26 \%$ and $17 \%$ in $8 \mathrm{~h}$. MeSeA treatment resulted in a better migration inhibitory effect than SeMet or MeSeCys. Of these, the migration inhibition of MeSeA and MeSeCys treatment in 4 $\mathrm{h}$ and $8 \mathrm{~h}$ was with statistical significance $(p<0.05)$. SeMet treatment resulted in a statistically significant inhibition of migration in $8 \mathrm{~h}(p<0.05)$ (Figure 4A). Compared to MeSeA treatment alone, MeSeA plus SAM significantly suppressed the migration of HeLa cells about $50 \%$ at $4 \mathrm{~h}$, which was statistically significant $(p<0.05)$ (Figure 4B). SeMet in the presence of SAM had no obvious effects on HeLa cells migration (Figure 4C). MeSeCys plus SAM also significantly suppressed the migration of HeLa cells compared to MeSeCys treatment alone, with statistical significance at $8 \mathrm{~h}(p<0.05)$ (Figure 4D). These data indicate that $\mathrm{MeSeA}$ and $\mathrm{MeSeCys}$ could inhibit the migration of tumor cells, which can be enhanced by SAM. 
A

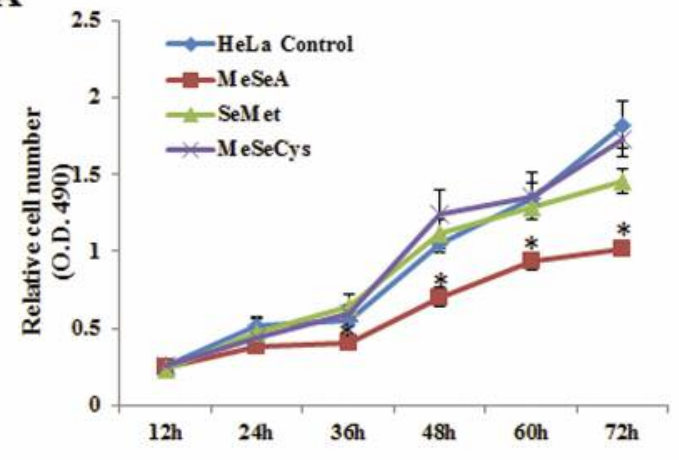

C

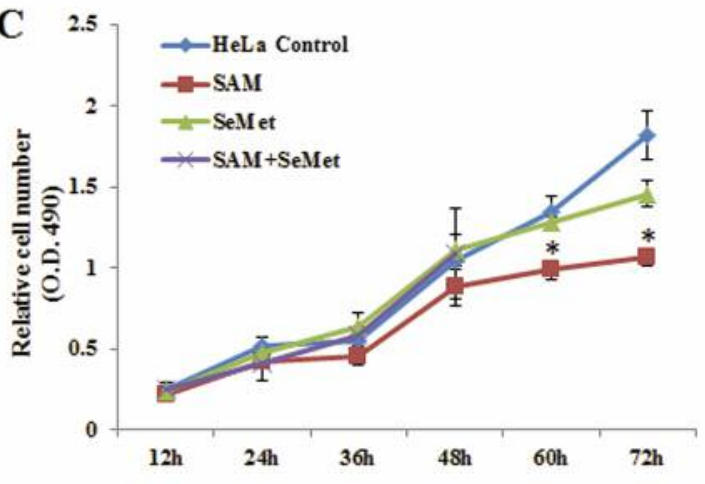

B

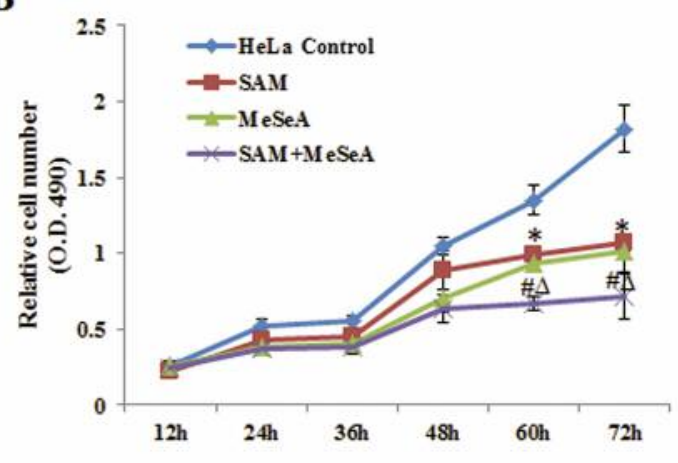

D

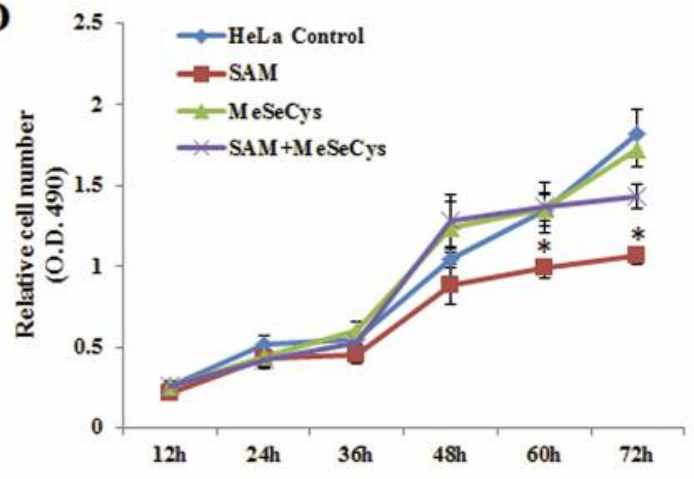

*VS. HeLa Control. $* P<0.05$

\#VS. SAM. $\# P<0.05$

$\triangle$ VS. without SAM. $\triangle P<0.05$

Figure 3. Selenium compounds and SAM synergistically inhibit the proliferation of HeLa cells. HeLa cells were treated with $3 \mu \mathrm{M}$ of selenium compounds (MeSeA, MeSeCys and SeMet) and/or $3 \mu \mathrm{M}$ of SAM for 12, 24, 36, 48, 60 and $72 \mathrm{~h}$, respectively. HeLa cells without treatment served as control group. MTT assay was used to detect the proliferation of HeLa cells. (A) Compared to HeLa control cells, MeSeA-treated group significantly inhibited the proliferation of HeLa cells at 36, 48, 60 and $72 \mathrm{~h}$ individually. (B) Compared with MeSeA or SAM alone, MeSeA combining SAM significantly suppressed HeLa cell proliferation, with statistical significance at 60 and $72 \mathrm{~h}$ individually. (C) SeMet in the presence or absence of SAM had no obvious effects on HeLa cells proliferation. (D) MeSeCys in the presence or absence of SAM had no obvious effects on HeLa cells proliferation. The results shown are representative of three individual experiments. Data are mean \pm S.D. $* p<0.05$ vs. HeLa control. \#p $<0.05 v s$. SAM. $\Delta p<0.05$ vs. without SAM.

Selenium compounds and SAM synergistically inhibit the adhesion of HeLa cells. In vitro Matrigel adhesion assay was used to determine the effects of selenium compounds and SAM on the adhesion of HeLa cells. Results are shown in Figure 5. Compared to HeLa control cells, the treatment of selenium compounds of MeSeA, SeMet or MeSeCys all inhibited the adhesion of HeLa cells $(* * p<0.01)$. The adhesion inhibition rate of MeSeA, SeMet or MeSeCys was $40 \%, 25 \%$ and $50 \%$, respectively. MeSeA and MeSeCys treatment resulted in a better adhesion inhibitory effect than SeMet. The combination of SAM with MeSeA (or SeMet) led to a significantly enhanced inhibition of adhesion in HeLa cells compared with MeSeA (or SeMet) or SAM treatment alone, with statistical significance $(\Delta p<0.05$,
$\# \# p<0.01)$. Compared to SAM treatment alone, MeSeCys plus SAM also significantly enhanced the adhesion inhibition of HeLa cells, with statistical significance $(\# \# p<0.01)$. These data indicated that MeSeA, SeMet, MeSeCys could inhibit the adhesion of tumor cells, which can be partly enhanced by SAM.

\section{Discussion}

Recently selenium compounds have been shown to inhibit cell growth and induce apoptosis in a variety of human cancer cells in vitro. The antitumor activities of selenium compounds are dependent on their dose and chemical form. In this study, we found that $3 \mu \mathrm{M}$ of MeSeA obtained the 

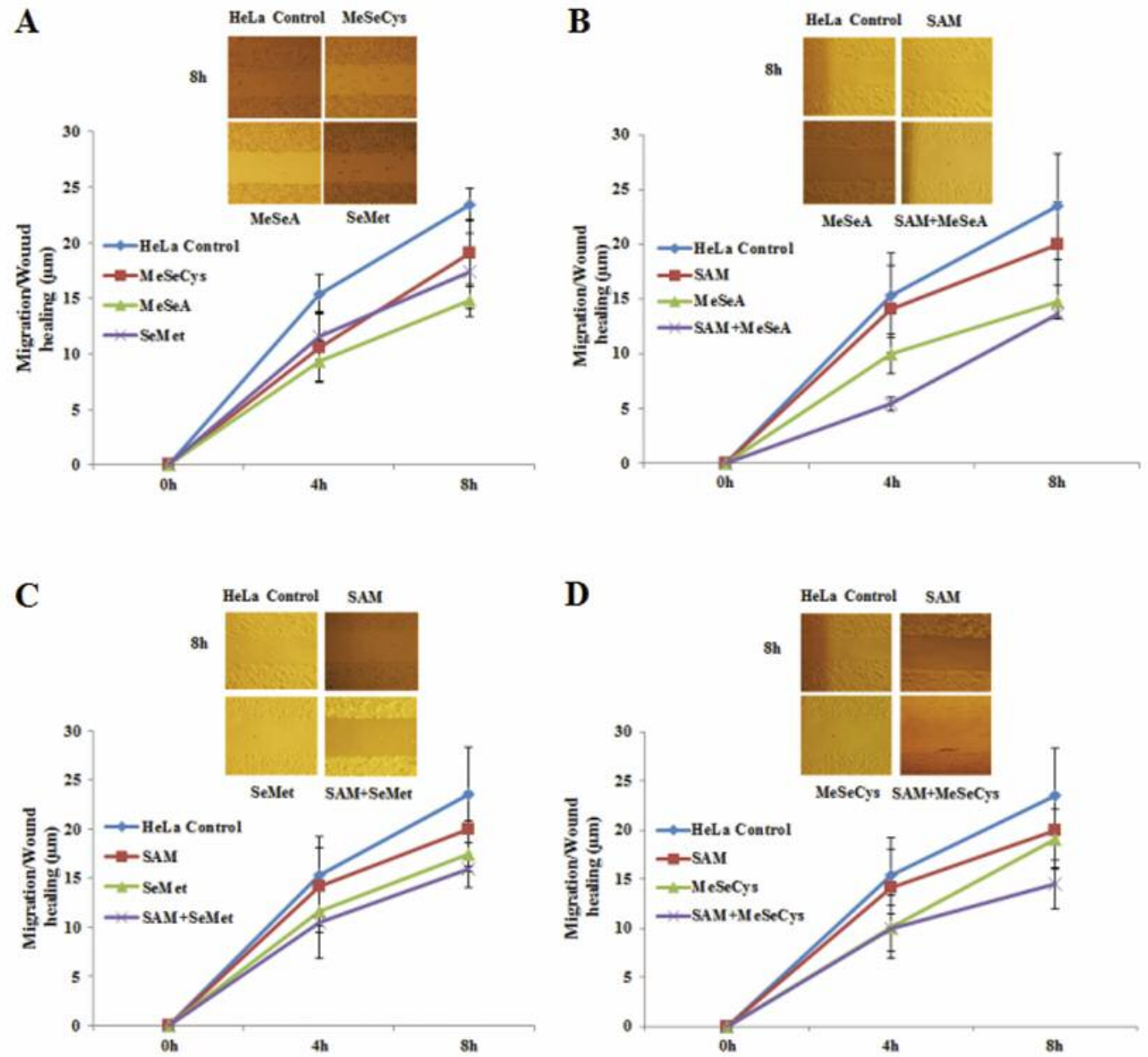

Figure 4. Selenium compounds and SAM alone or synergistically inhibit the migration of HeLa cells. Cells were seeded at a density of $2 \times 10^{5}$ cells/well into a 24-well plate, added with $3 \mu \mathrm{M}$ selenium compounds and/or SAM. The layer of cells was then scraped to create a wound. The movement of cells to heal the wound was recorded and analyzed using a time-lapsed video system. Cell migration was measured using Image $J$ analysis software. (A) The treatment of MeSeA, SeMet or MeSeCys all inhibited the migration of HeLa cells. MeSeA treatment resulted in better migration inhibitory effect than SeMet or MeSeCys. (B) MeSeA plus SAM significantly suppressed the migration of HeLa cells compared with MeSeA treatment alone. (C) SeMet in the presence or absence of SAM had no obvious effects on HeLa cells migration. (D) MeSeCys plus SAM also significantly suppressed the migration of HeLa cells compared with MeSeCys treatment alone for $8 \mathrm{~h}$. The results shown are representative of three individual experiments. Data are mean \pm S.D.

maximum proliferation-inhibiting effects compared to all other doses tested. Further study showed that MeSeA significantly inhibited ERK and AKT activation and the proliferation, migration and adhesion of HeLa cells, exhibiting better antitumor efficacy. MeSeCys partly inhibited the migration and adhesion of HeLa cells. SeMet showed weak antitumor effects in HeLa cells compared to $\mathrm{MeSeA}$ and MeSeCys. Our results were consistent with the previous report that MeSeA demonstrated superior in vivo growth inhibition compared to SeMet (5). The reason that selenium compounds exert different antitumor effects may be attributed to the different in vivo metabolic pathways of selenium compounds. Methylselenol is the key active antitumor substance of selenium compounds in vivo (22). In vivo, $\mathrm{MeSe} \mathrm{A}$ and $\mathrm{MeSeCys}$ can directly produce methylselenol by $\beta$-lyse and a reduction reaction $(23,24)$. Therefore, MeSeA and MeSeCys exhibit good antitumor effects $(5,8)$. The main metabolic product of SeMet in vivo is hydrogen selenide, which further generates selenoproteins, exhibiting the nutrition role of selenium (25). Only a small amount of SeMet can transform into methylselenol, exhibiting antitumor effects. Thus, to generate more methylselenol is the key of SeMet exerting antitumor roles. 


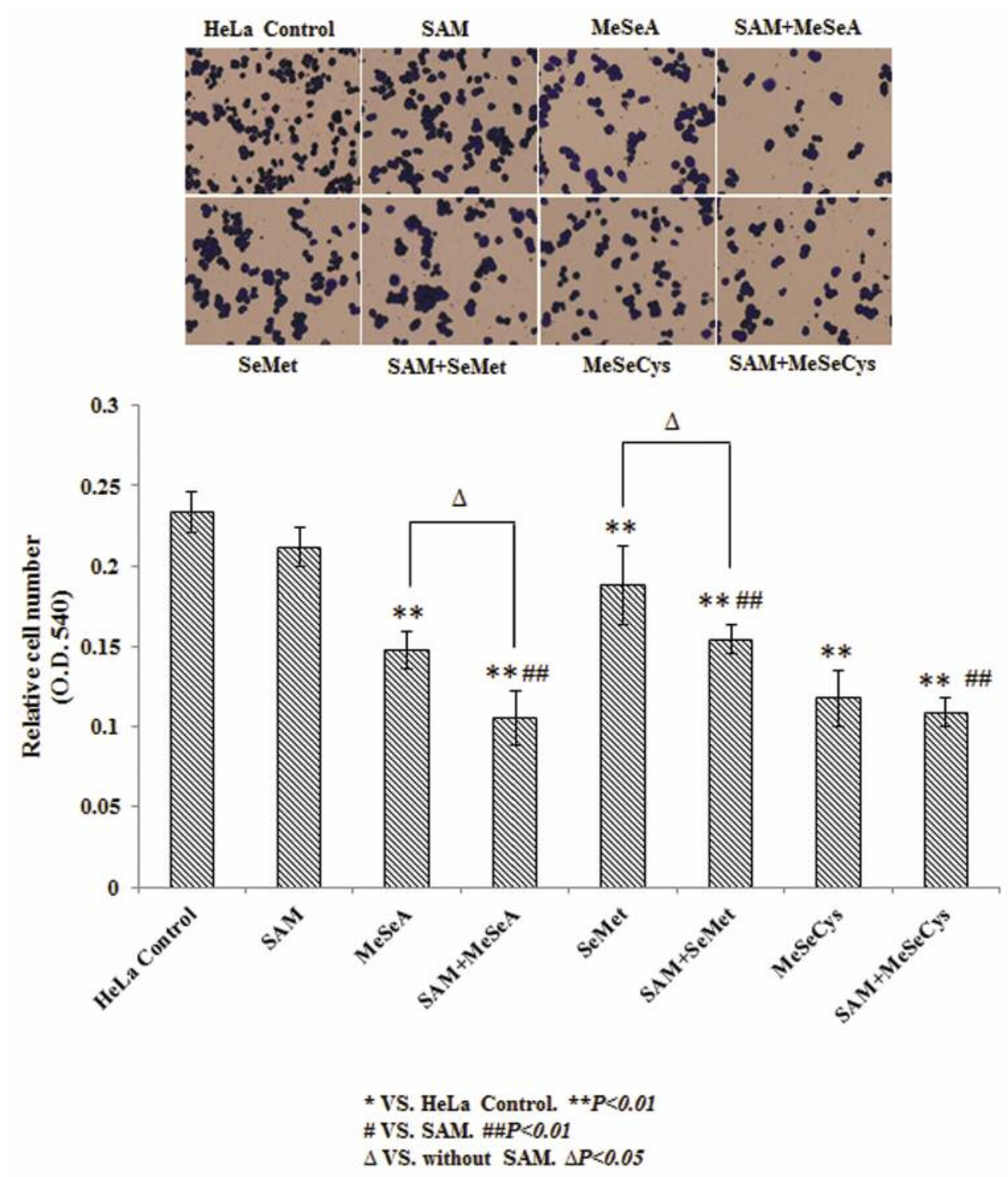

Figure 5. Selenium compounds and SAM synergistically inhibit the adhesion of HeLa cells. $4.5 \times 10^{4}$ HeLa cells were seeded onto the Matrigel basement membrane in normal medium and incubated. Then, adherent cells were fixed and stained in $0.5 \%$ crystal violet solution for 5 min. Cell density was determined by measuring the absorbance at $540 \mathrm{~nm}$ on a microplate reader. The results shown are representative of three individual experiments. Data are mean $\pm S . D .{ }^{*} p<0.05$, ${ }^{* *} p<0.01$ vs. HeLa control. \#p<0.05, \#\#p<0.01vs. SAM. $\Delta, p<0.05$ vs. without SAM.

SAM is synthesized in vivo using methionine and ATP as substrates, catalyzed by methionine adenosyltransferase (MAT). SAM participates in many biochemical reactions in human body including transmethylation and transsulfuration. In this study, the combination of SAM with MeSeA significantly suppressed the proliferation, migration and adhesion of HeLa cells compared to MeSeA alone. SAM plus MeSeCys partly suppressed the migration of HeLa cells compared to MeSeCys alone. SAM plus SeMet inhibited the adhesion of HeLa cells compared to SAM alone. These results show the synergistic effects of SAM and selenium compounds on HeLa cells tumor function. The difference seen in the synergistic effects of SAM maybe depends on the participation of SAM into the metabolism of the selenium intermediary metabolite. On SeMet metabolism pathways, SAM can methylate intermediary metabolite hydrogen selenide into methylselenol, exerting antitumor activity. On the metabolic pathways of MeSeA and MeSeCys, SAM can promote the inter-conversion of the intermediary metabolite methylselenol and two methyl selenium. Thus, the changes in the metabolic pathways lead to differential biological functions of selenium compounds. 
SAM has been widely used in humans as a dietary supplement with antidepressant and antioxidant properties. Oral administration of SAM to mice attenuates liver injury. But the influence of SAM on tumor function remains largely unknown. As a methyl donor in vivo, SAM makes some oncogenes methylated and inactive, further inhibiting corresponding tumor function. Our results demonstrated that SAM can synergistically enhance the antitumor efficacy of MeSeA in HeLa cells, suggesting a new strategy of treatment of high grade cervical cancer.

In summary, our data support a dose-dependent inhibition of human cervical cancer HeLa cells growth in vitro by MeSeA. MeSeA exhibits better tumor-suppressing effects on the proliferation, migration and adhesion of tumor cells than SeMet and MeSeCys. The combination of MeSeA with SAM significantly inhibited ERK and AKT activation, suppressed the proliferation, migration and adhesion of HeLa cells compared to MeSeA alone. Our study reveals the differences in the antitumor effects of selenium compounds, clarifies their molecular mechanism of action, and determines the role of SAM, which provided new insights into the synergistic tumor-inhibiting role of selenium compounds and SAM.

\section{Conflicts of Interest}

The Authors declare no conflicts of interest.

\section{Acknowledgements}

This work was supported by the National Natural Science Foundation of the People's Republic of China (No. 81372989) and the Beijing Natural Science Foundation (No. 7172144).

\section{References}

1 Whanger PD: Selenium and its relationship to cancer: an update. Br J Nutr 91: 11-28, 2004.

2 Ghadi FE, Ghara AR, Bhattacharyya $S$ and Dhawan DK: Selenium as a chemopreventive agent in experimentally induced colon carcinogenesis. World J Gastrointest Oncol 1: 74-81, 2009.

3 Hurst R, Hooper L, Norat T, Lau R, Aune D, Greenwood DC, Vieira R, Collings R, Harvey LJ, Sterne JA, Beynon R, Savovic $\mathrm{J}$ and Fairweather-Tait SJ: Selenium and prostate cancer: systematic review and meta-analysis. Am J Clin Nutr 96: 111122, 2012.

4 Amaral AF, Cantor KP, Silverman DT and Malats N: Selenium and bladder cancer risk: a meta-analysis. Cancer Epidemiol Biomarkers Prev 19: 2407-2415, 2010.

5 Li GX, Lee HJ, Wang Z, Hu H, Liao JD, Watts JC, Combs GF Jr and Lü J: Superior in vivo inhibitory efficacy of methylseleninic acid against human prostate cancer over selenomethionine or selenite. Carcinogenesis 29: 1005-1012, 2008.

6 Johnson WD, Morrissey RL, Kapetanovic I, Crowell JA and McCormick DL: Subchronic oral toxicity studies of Semethylselenocysteine, an organoselenium compound for breast cancer prevention. Food Chem Toxicol 46: 1068-1078, 2008.
7 Wang J, Jiao NL and Zheng J: Effects of Se-methylselenocysteine on biological behavior of and matrix metalloproteinase-2 expression in human breast cancer MDA-MB-231 cells. Ai Zheng 27: 119-125, 2008.

8 Bhattacharya A, Tóth K, Sen A, Seshadri M, Cao S, Durrani FA, Faber E, Repasky EA and Rustum YM: Inhibition of colon cancer growth by methylselenocysteine-induced angiogenic chemomodulation is influenced by histologic characteristics of the tumor. Clin Colorectal Cancer 8: 155-162, 2009.

9 Lippman SM, Klein EA, Goodman PJ, Lucia MS, Thompson IM, Ford LG, Parnes HL, Minasian LM, Gaziano JM, Hartline JA, Parsons JK, Bearden JD 3rd, Crawford ED, Goodman GE, Claudio J, Winquist E, Cook ED, Karp DD, Walther P, Lieber MM, Kristal AR, Darke AK, Arnold KB, Ganz PA, Santella RM, Albanes D, Taylor PR, Probstfield JL, Jagpal TJ, Crowley JJ, Meyskens FL Jr, Baker LH and Coltman CA Jr.: Effect of selenium and vitamin E on risk of prostate cancer and other cancers: the Selenium and Vitamin E Cancer Prevention Trial (SELECT). JAMA 301: 39-51, 2009.

10 Limburg PJ, Wei W, Ahnen DJ, Qiao Y, Hawk ET, Wang G, Giffen CA, Wang G, Roth MJ, Lu N, Korn EL, Ma Y, Caldwell KL, Dong Z, Taylor PR and Dawsey SM: Randomized, placebocontrolled, esophageal squamous cell cancer chemoprevention trial of selenomethionine and celecoxib. Gastroenterology 129: 863-873, 2005.

11 Zhao R, Domann FE and Zhong W: Apoptosis induced by selenomethionine and methioninase is superoxide mediated and p53 dependent in human prostate cancer cells. Mol Cancer Ther 5: 3275-3284, 2006.

12 Suzuki M, Endo M, Shinohara F, Echigo S and Rikiishi H: Rapamycin suppresses ROS-dependent apoptosis caused by selenomethionine in A549 lung carcinoma cells. Cancer Chemother Pharmacol 67: 1129-1136, 2011.

13 Goulet AC, Chigbrow M, Frisk P and Nelson MA: Selenomethionine induces sustained ERK phosphorylation leading to cell-cycle arrest in human colon cancer cells. Carcinogenesis 26: 109-117, 2005.

14 Verma A, Atten MJ, Attar BM and Holian O: Selenomethionine stimulates MAPK (ERK) phosphorylation, protein oxidation, and DNA synthesis in gastric cancer cells. Nutr Cancer 49: 184-190, 2004.

15 Mischoulon D and Fava M: Role of S-adenosyl-L-methionine in the treatment of depression: a review of the evidence. Am J Clin Nutr 76: 1158S-1161S, 2002.

16 Kim J, Lee EY, Koh EM, Cha HS, Yoo B, Lee CK, Lee YJ, Ryu $\mathrm{H}$, Lee KH and Song YW: Comparative clinical trial of Sadenosylmethionine versus nabumetone for the treatment of knee osteoarthritis: an 8-week, multicenter, randomized, double-blind, double-dummy, Phase IV study in Korean patients. Clin Ther 31: 2860-2872, 2009.

17 Módis K, Coletta C, Asimakopoulou A, Szczesny B and Chao C, Papapetropoulos A, Hellmich MR and Szabo C: Effect of Sadenosyl-L-methionine (SAM), an allosteric activator of cystathionine- $\beta$-synthase (CBS) on colorectal cancer cell proliferation and bioenergetics in vitro. Nitric Oxide 41: 146156, 2014.

18 Guruswamy S, Swamy MV, Choi CI, Steele VE and Rao CV: Sadenosyl L-methionine inhibits azoxymethane-induced colonic aberrant crypt foci in F344 rats and suppresses human colon cancer Caco-2 cell growth in 3D culture. Int J Cancer 122: 2530,2008 . 
19 Ham MS, Lee JK and Kim KC: S-adenosyl methionine specifically protects the anticancer effect of 5-FU via DNMTs expression in human A549 lung cancer cells. Mol Clin Oncol 1: 373-378, 2013.

20 Parashar S, Cheishvili D, Arakelian A, Hussain Z, Tanvir I, Khan HA, Szyf M and Rabbani SA: S-adenosylmethionine blocks osteosarcoma cells proliferation and invasion in vitro and tumor metastasis in vivo: therapeutic and diagnostic clinical applications. Cancer Med 4: 732-744, 2015.

21 Fernandes AP, Wallenberg M, Gandin V, Misra S, Tisato F, Marzano C, Rigobello MP, Kumar S and Björnstedt $\mathrm{M}$ : Methylselenol formed by spontaneous methylation of selenide is a superior selenium substrate to the thioredoxin and glutaredoxin systems. PLoS One 7: e50727, 2012.

22 Ip C, Thompson HJ, Zhu Z and Ganther HE: In vitro and in vivo studies of methylseleninic acid: evidence that a monomethylated selenium metabolite is critical for cancer chemoprevention. Cancer Res 60: 2882-2886, 2000
23 Navarro-Alarcon M and Cabrera-Vique C: Selenium in food and the human body: A review. Sci Total Environ 400: 115-141, 2008.

24 Suzuki KT, Tsuji Y, Ohta Y and Suzuki N: Preferential organ distribution of methylselenol source Se-methylselenocysteine relative to methylseleninic acid. Toxicol Appl Pharmacol 227: 76-83, 2008.

25 Mangiapane E, Pessione A and Pessione E: Selenium and selenoproteins: an overview on different biological systems. Curr Protein Pept Sci 15: 598-607, 2014.
Received May 19, 2017

Revised June 9, 2017

Accepted June 12, 2017 